\title{
Report of the EMI Testing of the Johnson Noise Thermometry System
}

\section{June 2014}

Prepared by

N. Dianne Bull Ezell Charles L. Britton Jr. Michael Roberts

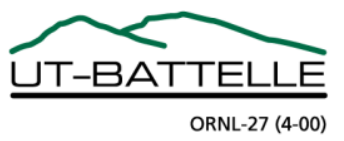




\title{
DOCUMENT AVAILABILITY
}

Reports produced after January 1, 1996, are generally available free via the U.S. Department of Energy (DOE) Information Bridge.

Web site http://www.osti.gov/bridge

Reports produced before January 1, 1996, may be purchased by members of the public from the following source.

\author{
National Technical Information Service \\ 5285 Port Royal Road \\ Springfield, VA 22161 \\ Telephone 703-605-6000 (1-800-553-6847) \\ TDD 703-487-4639 \\ Fax 703-605-6900 \\ E-mail info@ntis.gov \\ Web site http://www.ntis.gov/support/ordernowabout.htm
}

Reports are available to DOE employees, DOE contractors, Energy Technology Data Exchange (ETDE) representatives, and International Nuclear Information System (INIS) representatives from the following source.

Office of Scientific and Technical Information

P.O. Box 62

Oak Ridge, TN 37831

Telephone 865-576-8401

Fax 865-576-5728

E-mail reports@osti.gov

Web site http://www.osti.gov/contact.html

This report was prepared as an account of work sponsored by an agency of the United States Government. Neither the United States Government nor any agency thereof, nor any of their employees, makes any warranty, express or implied, or assumes any legal liability or responsibility for the accuracy, completeness, or usefulness of any information, apparatus, product, or process disclosed, or represents that its use would not infringe privately owned rights. Reference herein to any specific commercial product, process, or service by trade name, trademark, manufacturer, or otherwise, does not necessarily constitute or imply its endorsement, recommendation, or favoring by the United States Government or any agency thereof. The views and opinions of authors expressed herein do not necessarily state or reflect those of the United States Government or any agency thereof. 


\title{
REPORT OF THE EMI TESTING OF THE JOHNSON NOISE THERMOMETRY SYSTEM
}

\author{
Author(s) \\ N. Dianne Bull Ezell \\ Charles L. Britton Jr \\ Michael Roberts
}

Date Published: June 2014

\author{
Prepared by \\ OAK RIDGE NATIONAL LABORATORY \\ Oak Ridge, Tennessee 37831-6283 \\ managed by \\ UT-BATTELLE, LLC \\ for the \\ U.S. DEPARTMENT OF ENERGY \\ under contract DE-AC05-00OR22725
}




\section{CONTENTS}

\section{Page}

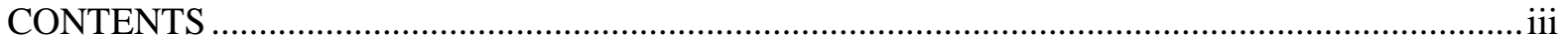

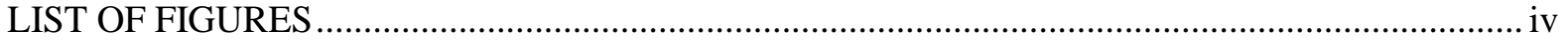

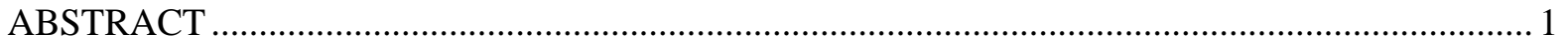

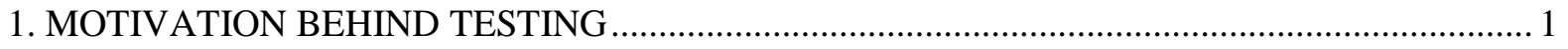

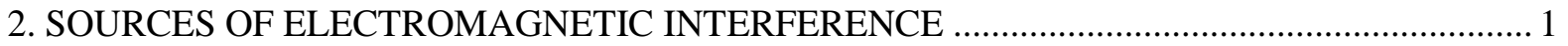

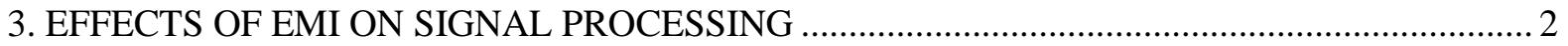

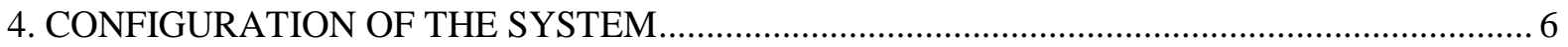

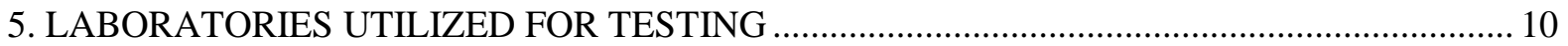

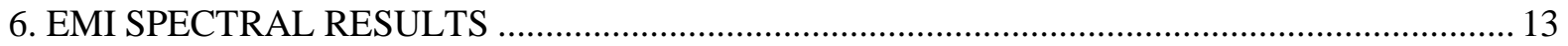

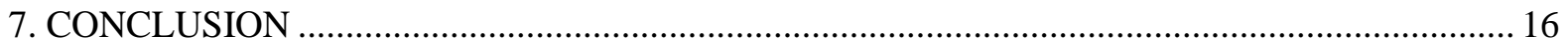

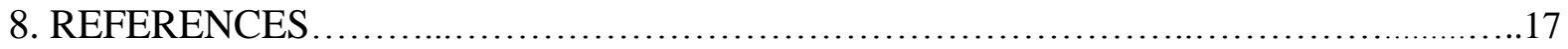




\section{LIST OF FIGURES}

Figure

Page

$1 \quad$ Random Noise with Transient EMI

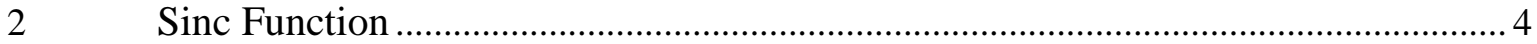

$3 \quad$ Windowing Options (Time and Frequency Domains) ….......................................5

$4 \quad$ JNT System Block Diagram .......................................................................6

$5 \quad$ JNT System Photographs .......................................................................... 9

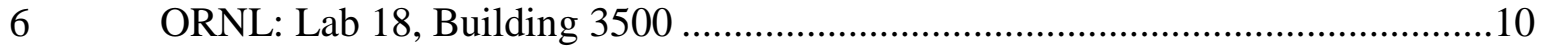

$7 \quad$ Average Power Spectral Density in Lab 18- Spikes Represent Noise Sources .....10

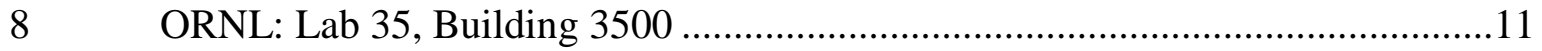

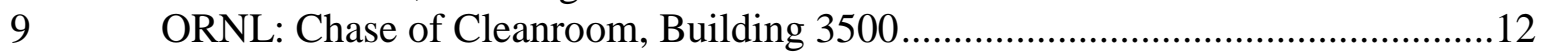

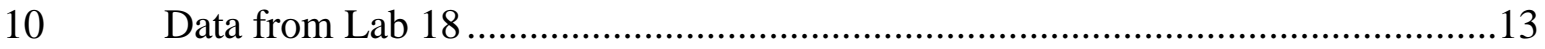

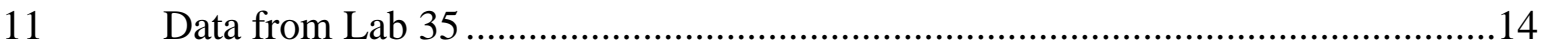

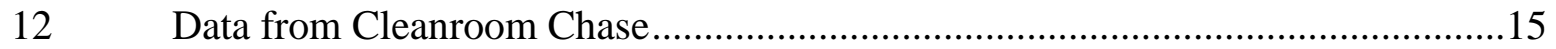




\begin{abstract}
This report summarizes the Electromagnetic Interference (EMI) testing of the Johnson Noise Thermometry System developed at ORNL. The EMI performance is very important for Johnson Noise Thermometry because it requires accurate measurement of a very small noise signal that is amplified 10,000 times. Any interference in the form on pickup from external signal sources from such as fluorescent lighting ballasts, motors, etc. can skew the measurement. Testing is therefore very important in determining the effects of these external noise sources. Results from testing in several environments with various sources of EMI are presented here.
\end{abstract}

\title{
1. MOTIVATION BEHIND TESTING
}

There are several reasons to perform EMI testing instead of simply employing the system in a prototypical environment. Among these are:

A. Functional testing - The basic functionality of the system is tested during development but is always subject to change while packaging is finalized, circuits are modified, etc. Running a continuous set of tests determines if the system can run day after day unattended without failure and functions as expected.

B. Dynamic Range testing - It is necessary to test the system under a wide variety of operational and EMI conditions to see if there are any issues with varying EMI.

C. Software testing - Long runs are essential in determining any issues with communications and signal processing algorithms.

D. System integrity - The system integrity test to find problems missed during design and fabrication is ongoing. Noise filtering is an issue on the incoming power, hardware failures such as capacitors on the interface board, and crosstalk in the pilot-tone wiring.

\section{SOURCES OF ELECTROMAGNETIC INTERFERENCE}

EMI is emitted from a variety of sources in both industrial and household environments. EMI arises from time-varying electric and magnetic fields present in almost every piece of electrical and electronic equipment on the planet. Among these interferers are:

A. Power Lines - The most ubiquitous interferer is the common $60-\mathrm{Hz}$ power line that is everywhere in buildings and outdoors. Power lines inside building act as antennas and carry the low-frequency power signals to every part of the indoor environment. Distortion of the AC waveform due to loading worsens the situation by creating harmonics of $60 \mathrm{~Hz}$ and higher frequencies. Additionally, when current is flowing in the lines, magnetic fields are generated around the lines and radiate outwards from the conductors. These can couple into circuits as well as the electric-field variations.

B. Lights - Electronic signals from fluorescent lights are a particularly nasty signal because the light ballasts are actually switching boost converters that oscillate (usually) above human hearing in the region around and above $20 \mathrm{kHz}$. These are easily picked up by sensitive equipment and can cause band interference of several $\mathrm{kHz}$ around the oscillation frequency.

C. Computer Power Supplies - The power supplies in almost all computers are also switching supplies that run anywhere from $30-50 \mathrm{kHz}$. They are very noisy due to a minimum amount of shielding around the supply (usually just enough shielding to pass FCC certification). These also cause problems with power lines in that the switching 
signals may not be well isolated from the input power and the high-frequency signals get transmitted back on the $120-\mathrm{V}$ power line.

D. Cathode-Ray Tubes - They are not as big a problem as they once were, but some legacy systems still use cathode-ray tubes. The vertical and horizontal sweep circuitry is a prime source of interference in the few tens of $\mathrm{kHz}$ region.

E. Cellphones - The internal oscillators of cellphone radio boards can be problematic. These cause intermodulation distortion that can be picked up.

F. Motors - Motors are a particular problem since they generate both electric and magnetic fields. Induction motors cause transients on power lines during startup. AC/DC motors with brushes cause large amounts of noise due to very high frequency noise of the brushes and armatures.

G. Data Lines (Ethernet) - Data lines behind walls and across floors and lab benches generate interference due to the currents and voltages within the lines.

It should be clear from this brief list that interference is everywhere in a normal environment. The key to building a working system in the environments likely to use JNT is to ensure the appropriate signal processing and filtering enables accurate measurements.

\section{EFFECTS OF EMI ON SIGNAL PROCESSING}

Electromagnetic interference (EMI) is any non-random signal from the environment in which the JNT system operates that is added to the noise voltage of the sensor through either a conduction or radiation mechanism. Most real sources of EMI can be classified as either transient or periodic. Transient EMI is a signal that begins, persists for some time and then stops. Periodic EMI is a signal that is always present and repeats a pattern indefinitely.

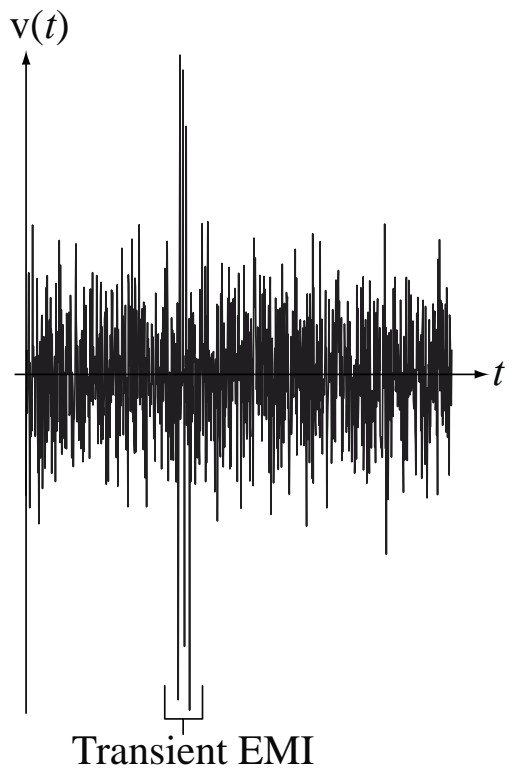

Fig. 1. Random Noise with Transient EMI

Transient EMI , similar to Fig. 1, can be detected as a significant deviation in the acquired signal from the expected noise voltage of the sensor. The sensor noise voltage is random so it is unknown exactly how it should appear, but it is known that its average value is zero and 
that it is Gaussian distributed. Therefore, the variations from zero should, with probability 0.997, lie within +/- 3 standard deviations and any signal beyond 6 standard deviations will be, with very high probability, transient EMI. The output channel signals are acquired by sampling at a rate $f_{s}$ greater than twice the practical bandwidth of the signals, according to the sampling theorem, to avoid significant aliasing. The samples are acquired in "blocks". One block has $N$ samples and a typical block size is $N=2^{18}$. The standard deviation is estimated from a block of samples and then all values of the signal in that block of samples are checked for any excursions that exceed 6 standard deviations. If such excursions are found we can "zero out" the transient EMI by multiplying the signal by zero over a range of time before, during and after the detected excursion, and keep the remainder of the samples as good data. The threshold of 6 standard deviations is arbitrary. It could be higher, in which case we run the risk of not detecting some transient EMI that does occur. It could also be lower, in which case we run the risk of rejecting actual random noise fluctuations by classifying them as transient EMI. Fortunately, in our experience the transient EMI observed in the testing has been very large and obvious and easily detected with a threshold of 6 standard deviations.

If the transient EMI persists for a significant fraction of the block time it may artificially inflate the estimated standard deviation. Also, the standard deviation of a block of samples is compared to the standard deviations of the last few previous blocks of samples to detect a block of samples with an abnormally high standard deviation. This is a valid check because, for physical reasons, the sensor temperature and, therefore, the standard deviation of the sensor noise voltage, cannot change quickly. If the transient EMI persists over multiple blocks of samples it can become, as a practical matter, undetectable because it distorts the estimates of standard deviation and is not possible to distinguish all of it from the sensor noise voltage. In most practical situations this does not happen but in a very high EMI environment it could happen.

Periodic EMI presents a different challenge to the signal processing. It is not possible to detect and reject it with the same methods used for transient EMI because it is always present and does not cause large sudden excursions of the signal above some threshold multiple of the standard deviation. But periodic EMI does have a characteristic that makes it detectable. A fundamental principle of Fourier analysis of signals is that the continuous-time Fourier transform (CTFT) of a continuous-time periodic signal consists only of impulses. That is, it is confined to a set of exact frequencies and the CTFT at all other frequencies is free of the effects of the periodic signal. So the software must detect periodic EMI using its Fourier transform; that is, in the "frequency domain" instead of in the "time domain" where it detects transient EMI.

The JNT software approximates the CTFT's of the amplifier/filter output voltages by computing the discrete Fourier transform (DFT) of a block of $N$ samples taken from each of them. The CTFT of a truly periodic signal consists only of impulses. The DFT of one block of samples taken from a periodic signal consists of "spikes" which are "sinc" functions, illustrated in Fig. 2, centered where the impulses of the CTFT would have been. 


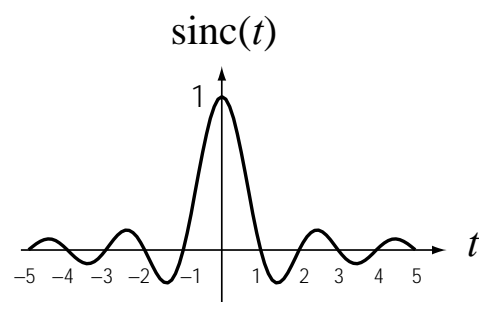

Fig. 2. Sinc Function

The exact shape of the spikes depends on the block size. Larger blocks make for taller and narrower sinc functions. As the block size approaches infinity these spikes approach impulses. The blocks are as long as is practically possible, given computer memory constraints, to make the spikes as close to impulses as possible, but they can never be true impulses. That means that the spikes have a finite height and non-zero width in the frequency domain. The computed power spectral density (PSD) of the sensor noise voltage is mathematically determined by knowledge of the two amplifier/filter output signals and the cross power frequency response of the amplifiers and filters that produce them. The exact shape is determined by how the estimated PSD of the sensor noise voltage is computed using the DFT, to approximate the true continuous-time PSD.

If a block of samples is acquired and computes its DFT directly, that is what is known in digital signal processing as "windowing" with a "rectangular window". That is, it is as though the original continuous-time signal is multiplied by a function whose value is one over the block time and zero elsewhere and therefore has a rectangular shape. As mentioned above, the effect in the frequency domain of this rectangular window is to replace the impulses of the CTFT with sinc functions in the DFT which peak at the EMI frequencies of the CTFT but also "ripple" out from the peak to other frequencies with a decreasing amplitude as the distance from the peak increases. This effect is called "spectral leakage". It takes some of the signal power at a periodic EMI frequency and spreads it out into adjacent frequencies and increases the apparent PSD of the computed sensor noise voltage. This is a source of error in estimating the PSD of the sensor noise voltage and therefore also in estimating the temperature of the sensor. The software can identify and ignore the large main peak caused by the periodic EMI but the extra ripples caused by spectral leakage are still there and contribute to temperature error.

If there is only one periodic EMI signal, the effects of spectral leakage can, in some special cases, be eliminated by choosing a sampling rate that makes the block time an exact integer multiple of the fundamental period of that single periodic EMI signal. If there is more than one EMI signal this technique will not work unless all the EMI signals have a finite common fundamental period. Usually, in practice, one source of EMI is the omnipresent (nominally) $60-\mathrm{Hz}$ power line frequency and its harmonics. If that is the only EMI signal then making the block time an integer multiple of the fundamental period of the line frequency would greatly reduce the leakage effects. Then, even when the line frequency changes slowly and slightly, the leakage effects would be mostly gone. Another way to reduce spectral leakage is to make the data blocks longer. But, as mentioned above, that is limited by the size of the memory in the computer dedicated to JNT signal processing. If the block size stays the same, the effects of spectral leakage can still be modified by using a window of a different shape (other than rectangular) that minimizes the spread of the signal 
power to adjacent frequencies. Some windowing options are demonstrated in Fig. 3. When this is done the central peak is wider but the side lobes are smaller. This reduces spectral leakage outside the central peak but spectral leakage can never be completely eliminated.

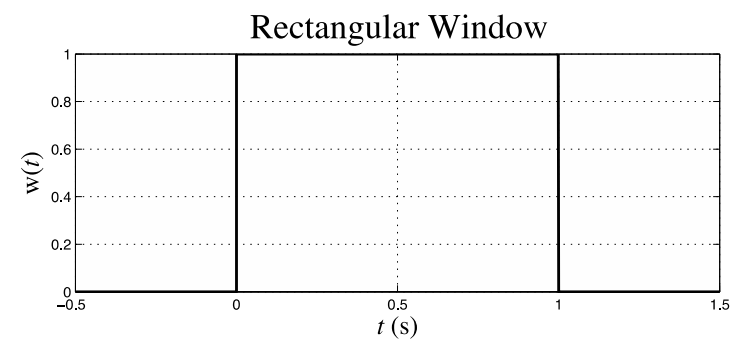

Blackman Window

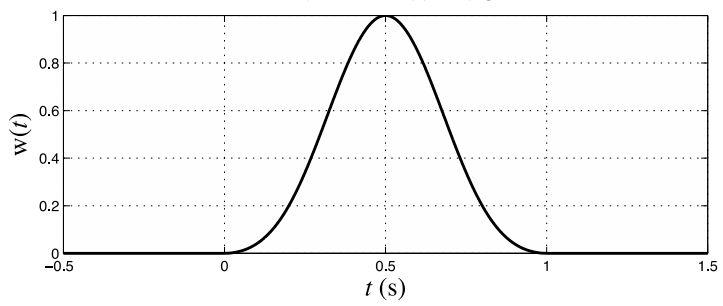

Flat Top Window

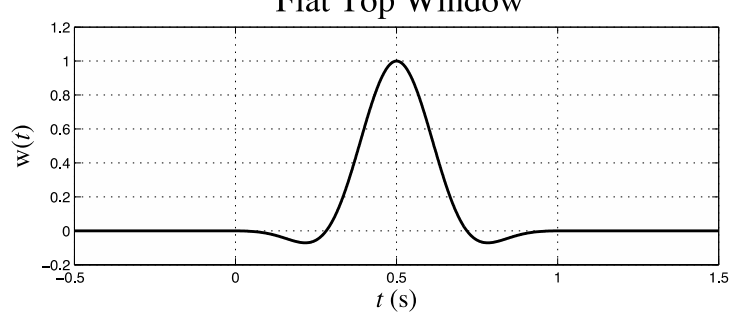

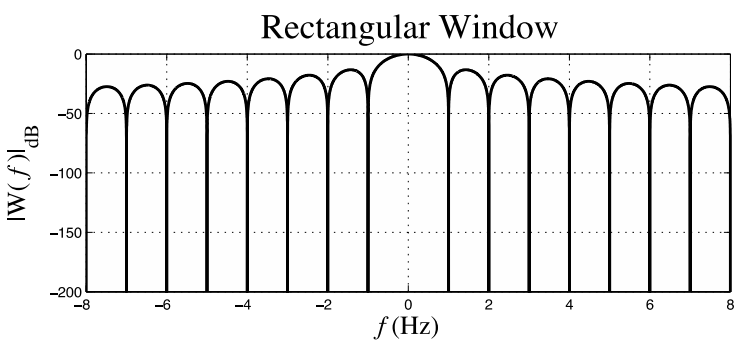

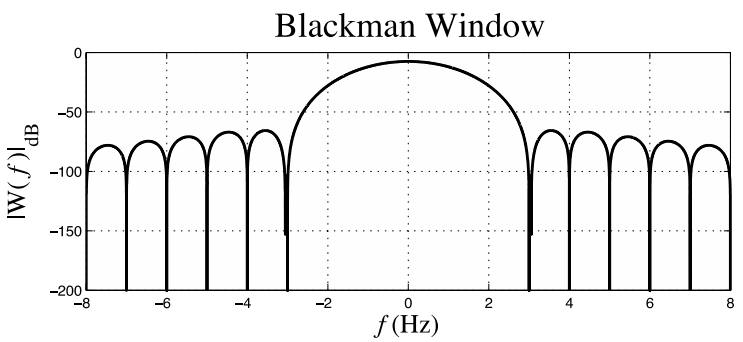

Flat Top Window

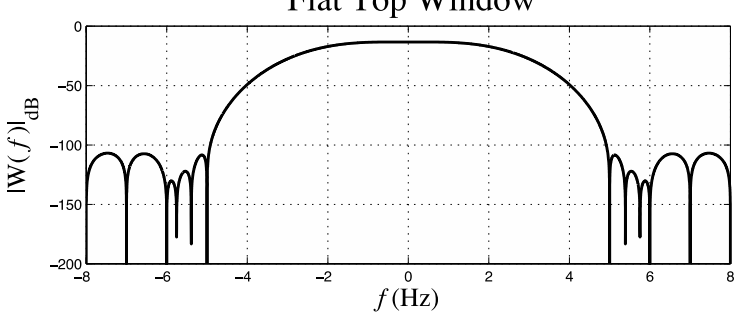

Fig. 3. Windowing Options (Time and Frequency Domains)

In the techniques used to detect and eliminate the effects of transient and periodic EMI, the common theme is the detection and rejection of unusual or non-typical signal behavior in either the time or frequency domain. In those extreme cases in which the EMI signal power is very large in comparison with the signal power of the desired signal the EMI may dominate the desired signals to such an extent that the EMI itself, in a sense, becomes the "normal" signal and cannot be eliminated. This determines a fundamental limit on the use of JNT for thermometry. In extreme EMI environments its accuracy becomes so poor as to render it unusable. 


\section{CONFIGURATION OF THE SYSTEM}

As presented in the previous reports [1-4], the JNT system consists of several parts, shown in Fig. 4. This report describes the final configuration of the JNT system and includes a brief description of each major system component.

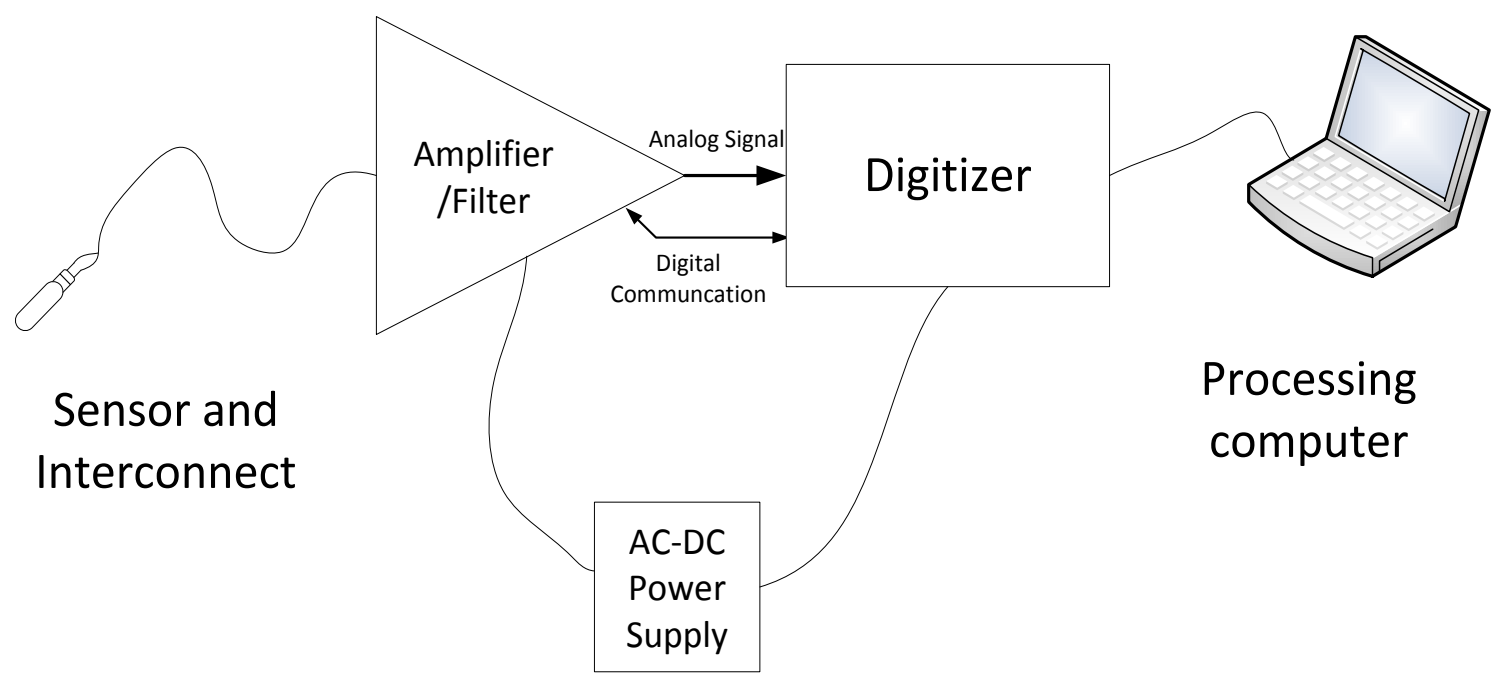

Fig. 4. JNT System Block Diagram

\section{Amplifier/Filter}

1. Preamplifier. The noise signal from the resistance thermometer detector (RTD) sensor is extremely small and needs to be sensed with an appropriate preamplifier having very low inherent noise and relatively high gain. The low noise is required so that the preamplifier injects the least possible amount of its own noise into the signal which would result in an incorrect reading. The gain is required so that the very small noise signal amplitude can be increased to a point where the electronic noise of successive stages will not be a significant source of error.

The RTD noise is sensed differentially by two independent channels which are treated as separate but equal amplifier chains. The signal at each of the two channel outputs contains primarily the sensor noise plus a small amount of noise from the filters and the other amplifiers associated with the channel. The final software processing of the signals includes routines to cross-correlate the noise between the two channels. The only signal of interest that is in both channels is the desired sensor noise (correlated) so that the cross-correlation of the two channels removes other electronic noise from the amplifiers and filters.

2. Differential Amplifier/Gain. The output of the differential preamplifier consists not only of the desired noise of the resistor but also the common-mode noise of extraneous pickup in the sensor lines. In order to remove the undesired pickup noise the differential preamplifier output is processed with a differential amplifier which passes the resistor signal of interest and cancels most of the pickup noise.

3. Bandpass Filter/Gain. The bandpass filter design utilizes the Sallen-key filter topology to implement the high-pass and low-pass functions. The Sallen-Key (SK) 
filter was chosen for this application for two reasons. First, this filter topology utilizes a finite-gain operational amplifier configuration which maintains a stable transfer function over variations in operational amplifier performance thus simplifying the choice of amplifiers in the design. Second, the filter design is relatively simple since each second-order filter block is entirely independent of the other filter blocks. The filter and gain stages were discussed in more detail in previous reports.

4. Ohmmeter Front-End/Bandpass Filter. For the DC resistance measurement (ohmmeter), a $100-\mu \mathrm{A}$ current is supplied to the RTD through a low-noise configuration that has a minimal impact on the measured noise. Two amplifiers, one for each side of the RTD, measure the voltage drop across the RTD due to the known $100-\mu \mathrm{A}$ current being injected. The two amplifiers are input to a differencing amplifier (thus forming a differential amplifier) with gain and then filtered with a low-pass filter to remove high-frequency noise. This configuration removes common-mode offset and noise to enable an accurate DC measurement. Since this is a 4-wire measurement, the current is supplied through one set of wires to the RTD and the voltage is measured across the remaining set of wires.

\section{Digitizer}

The digitizer receives the processed analog inputs from the amplifier/filter module and translates them into digital information, which is then sent to the signal processing program residing on the processing computer. The waveform synthesizer is used to generate the pilot tone to the processing electronics. The external computer acts as the system controller, which programs and operates both the digitizer and the digital waveform synthesizer.

Because there are numerous commercial options for the ADCs and because of the limited time and budget associated with this project, a mostly commercial, off-the-shelf system was chosen for implementation. The individual modules are described below.

1. Data Acquisition System (DAQ) - (Agilent U2531A). The U2531A is the heart of the data-acquisition system. The device contains four 14-bit, 2-Msamples/s analogto-digital converters (ADCs). In addition, it contains TTL-programmable digital outputs, digital counter channels, and 2 analog output channels. The only functionality used in this application is the set of four ADCs. Two of the ADCs are used for signals from the noise channels, one is used for the DC ohmmeter signal, and the fourth handles the pilot-tone digitization. The device is programmed and controlled from a standard USB interface, which is connected through a USB hub to the control computer. To minimize internally-generated electromagnetic interference, the U2531A is wrapped in copper mesh and single-point grounded to the main chassis.

2. Pilot-Tone Generator - (Agilent 33220A Digital Waveform Generator). The pilot tone is a frequency-modulated sine wave that is swept from below $1 \mathrm{kHz}$ to about 130 $\mathrm{kHz}$ with very stable amplitude of approximately $0.75 \mathrm{~V}$ peak-peak. The Agilent $33220 \mathrm{~A}$ is used for this function, as it is capable of 14-bit, $50 \mathrm{ohm}$, 2-Msamples/s 
output. The device is also programmed and controlled from the USB interface, which is connected through a USB hub to the control computer. Again, to minimize internally-generated electromagnetic interference, the 33220A is also wrapped in copper mesh and single-point-grounded to the chassis.

3. Filter/Interface Board. The Filter/Interface Board (FIB) provides connections between the Amplifier Module and the rest of the system. The FIB's functions are as follows:

A. The two noise-channel outputs are fed from the Amplifier Module into two of the input channels of the Agilent U2531 through the FIB. The FIB provides an adjustable voltage gain of 2-20 and additional low-pass filtering, with cutoff frequencies of approximately $40 \mathrm{kHz}$. The DC voltage supply is taken from the input $\pm 15 \mathrm{~V}$ source and locally regulated to $\pm 12 \mathrm{~V}$.

B. The board also contains the circuitry to convert the single-ended output of the Agilent 33220A into an attenuated $(\sim 70 \mathrm{~dB})$ differential drive suitable for the Amplifier Module.

4. Computer Interface. The computer interface was chosen to be Universal Serial Bus (USB) for two reasons. First, consultation with local and industry colleagues indicated that for this prototype, the type of connectivity was not critical for the demonstration. Second, the most common interface for benchtop electronic equipment is USB due to its simplicity and ease of use. Consequently, a USB is used for the entire system. A USB hub was also installed in the box to enable convenient communication with the two Agilent modules, both of which use USB interfaces.

\section{AC/DC Power Supplies}

The power supplied to the digitizer system consists of clean DC voltage driver from two linear DC power supplies and 120V AC, all controlled by a single switch on the powersupply box (see Fig. 6). The Agilent U2531 is supplied from the factory with an external 12$\mathrm{V}$, 2-A switching supply which was, in earlier tests, found to inject noise into the Amplifier/Filter Module. To mitigate this for all possible configurations, we replaced the supply with a linear, non-switching DC supply and enclosed it in a $12 \times 10 \times 4$ " box with the system $+/ 15-\mathrm{V}$ supply.

\section{Processing Computer/ Interface Description}

The computer used for this system is a standard device running Windows ${ }^{\mathrm{TM}} 7$. Currently, the instrument system is running on a Dell desktop using LabView ${ }^{\mathrm{TM}}$ as the programming software for the JNT calculations and control; the plan is to remain with that platform combination through our present development/demonstration cycle. The machine is in a mini-tower configuration, which should be adequate for transporting to various sites for testing. The entire system is shown in Fig. 7 sitting on a portable cart. The steel box is on top, with the power-supply module on the bottom.

The system shown in Fig. 5 was constructed to have a minimal impact on present facilities utilizing the JNT system. To implement this, all of the electronics were integrated into a single front-end electronics steel box and all power supplies were integrated into a 
single power module. If an existing RTD in a facility was replaced with this system, the only added requirements would be a small amount of space for the boxes, a 120Vac power, and a USB cable to network with a computer. Previous reports describe fabrication and construction of individual components.

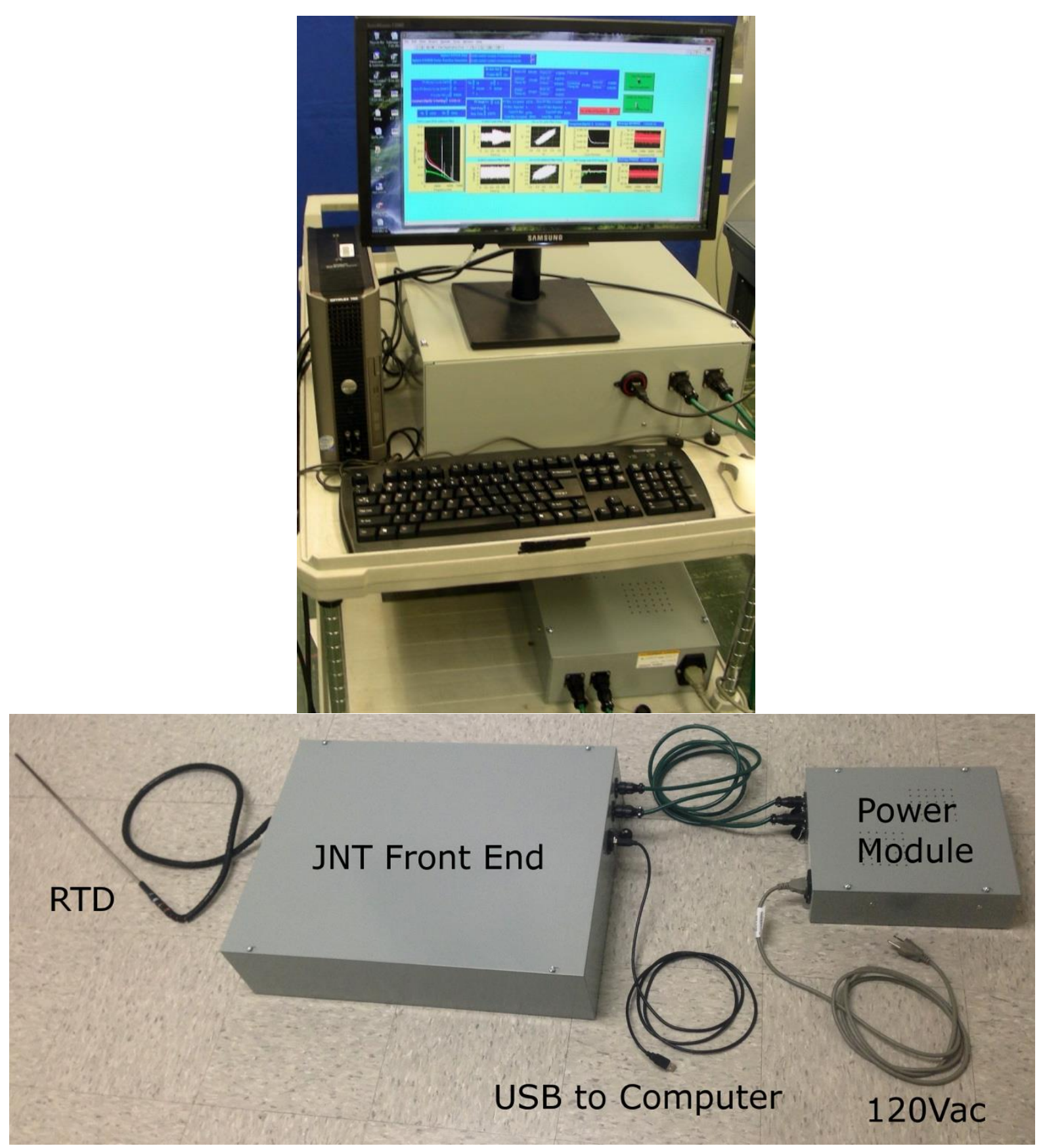

Fig. 5. JNT System Photographs 


\section{LABORATORIES UTILIZED FOR TESTING}

Two operating laboratories and an instrumented cleanroom chase were used for the initial EMI testing. These were chosen based on availability, accessibility to the environment, and similarity to JNT application environment. Also, in the case of the cleanroom chase, there were noise-generating motors. The labs were:

\section{$\underline{\mathrm{LAB}} 18$}

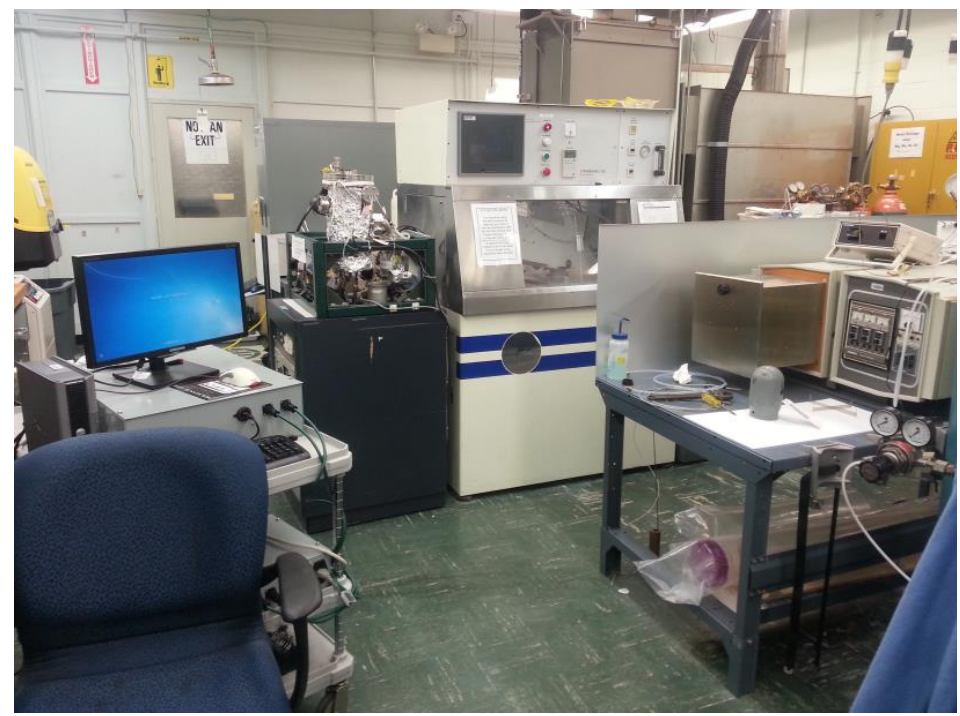

Fig. 6. ORNL: Lab 18, Building 3500

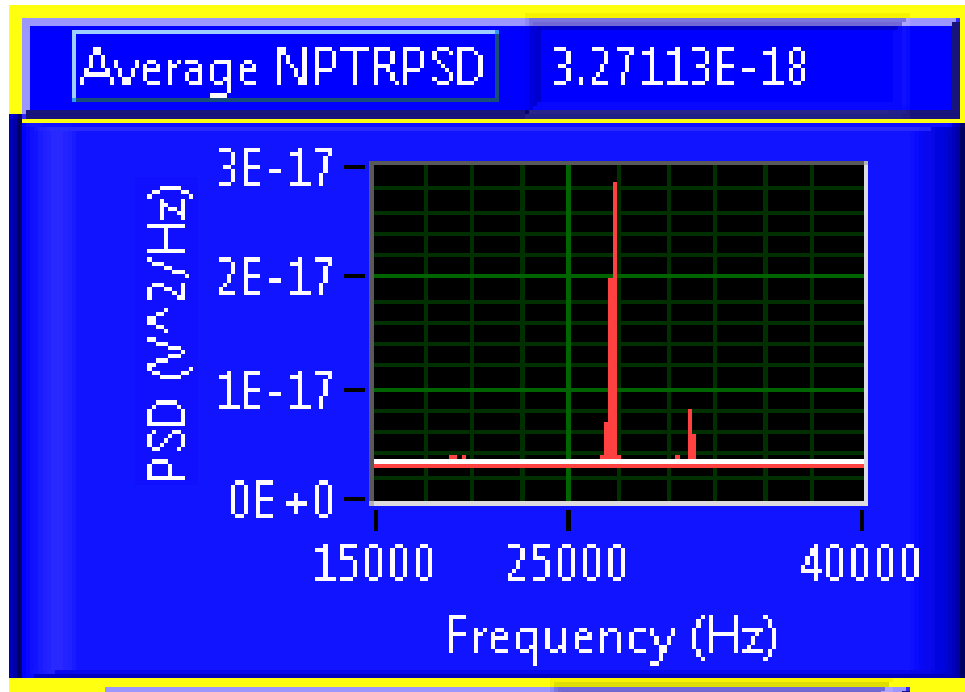

Fig. 7. Average Power Spectral Density in Lab 18- Spikes Represent Noise Sources

Lab 18 in building 3500, displayed in Fig. 6, has several mechanical vacuum pumps and hood ventilation motors. The lab also has various resistive furnaces. These are all possible noise and interference sources that can affect the JNT measurement. The largest noise source in this lab was the overhead fluorescent lamps at approximately $27 \mathrm{kHz}$, displayed in Fig. 7. 


\section{$\underline{\operatorname{LAB} 35}$}

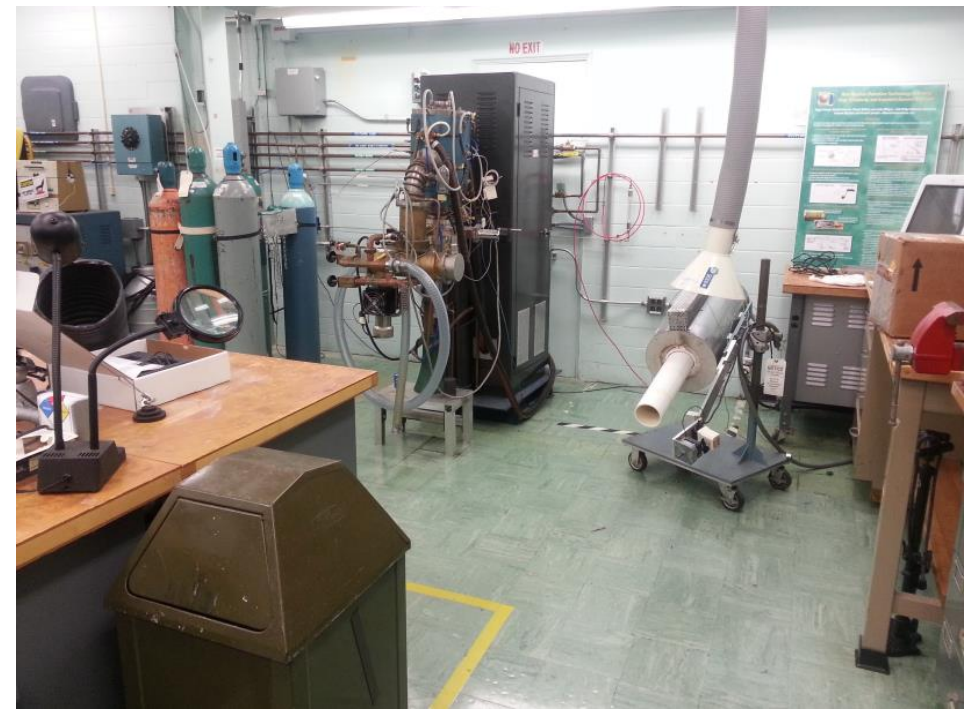

Fig. 8. ORNL: Lab 35, Building 3500

Lab 35 in building 3500, displayed in Fig. 8, is a somewhat quieter lab with no active pumps but in a different part of the building. The primary EMI source inherent in the lab is the fluorescent light ballasts.

\section{CLEANROOM CHASE}

The chase associated with the cleanroom in building 3500, displayed in Fig. 9, has numerous mechanical vacuum pumps, ventilation motors, electrical water pumps, RF power generators, and overhead fluorescent lamps. This environment is much noisier than the expected JNT application environment and therefore the chase test-environment enables testing of the systems limits. It can be concluded that if the system can survive this environment and still provide reasonable data, it will survive the application environment with successful results. 

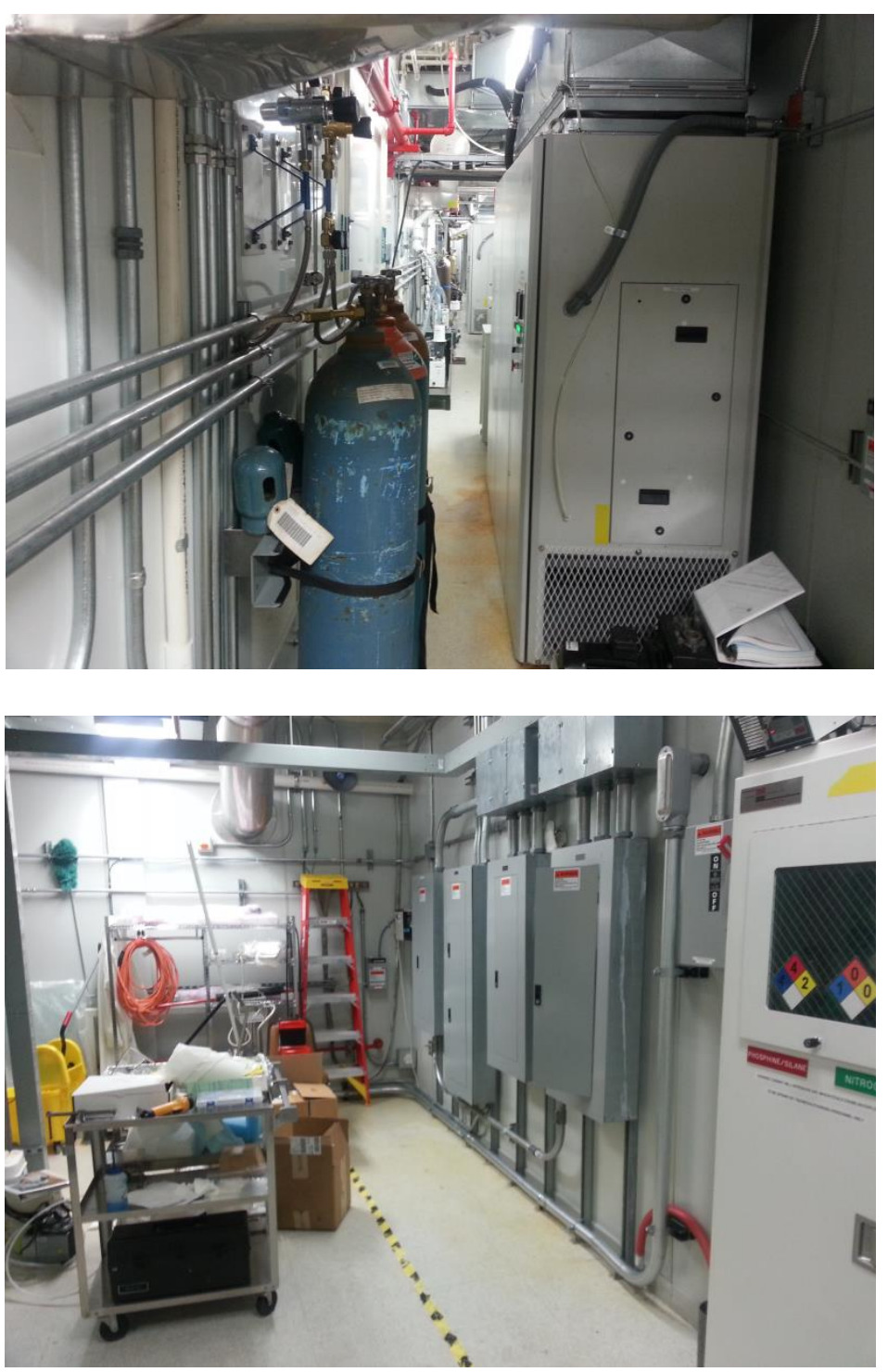

Fig. 9. ORNL: Chase of Cleanroom, Building 3500 


\section{EMI SPECTRAL RESULTS}

The EMI spectral results are presented as a summary of testing in each of the three environments. The data displayed in the following figures is the physical spectral measurements from the software. The data is raw spectrum out of each of the differential electronic channels (red and white) and the data after cross-correlation between the channels

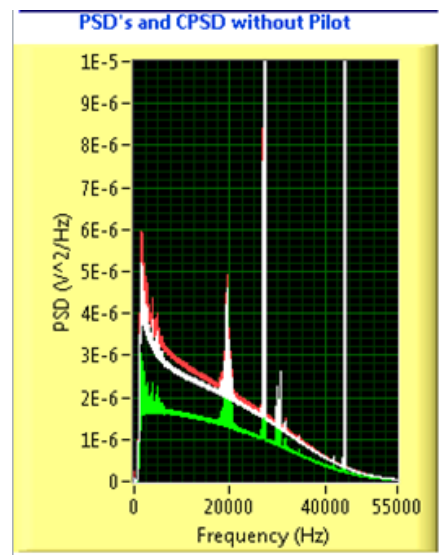

Cable $=0 \mathrm{~m}$, Error $=0 \mathrm{~K}$

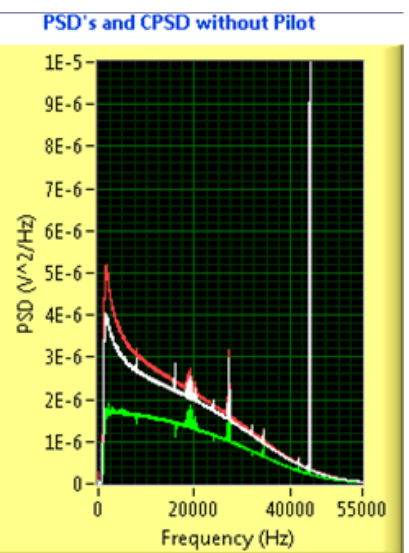

Cable $=5 \mathrm{~m}$, Error $=-0.67 \mathrm{~K}$

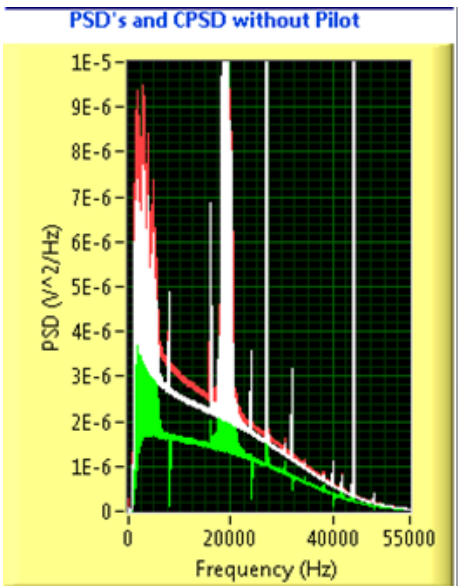

Cable $=10 \mathrm{~m}$, Error=1.4 K

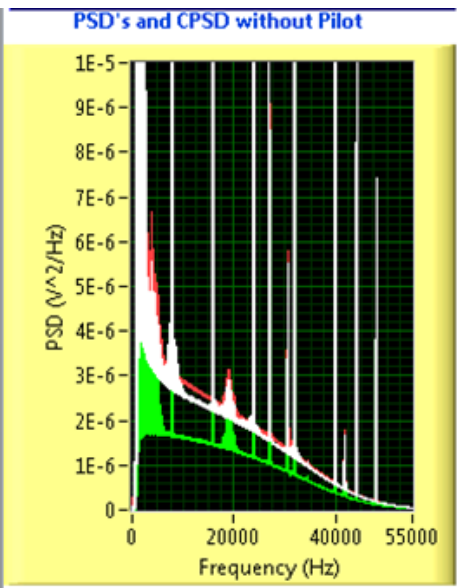

Cable $=25 \mathrm{~m}$, Error $=3.82 \mathrm{~K}$

Fig. 10. Data from Lab 18

(green). All of measurements utilize the same RTD. The data is grouped by cable length (in addition to the $1 \mathrm{~m}$ cable attached to the RTD) and the error relative to $0 \mathrm{~m}$ of cable. These are all room-temp measurements (approx. 25C), since the point of this testing is not trying to perform complete temperature runs but to evaluate the response due to EMI vs. cable length in different locations. In addition, there is no baseline recalibration between cable lengths to minimize time spent in the locations. In hindsight, this may have had a larger impact on the longest cable. 

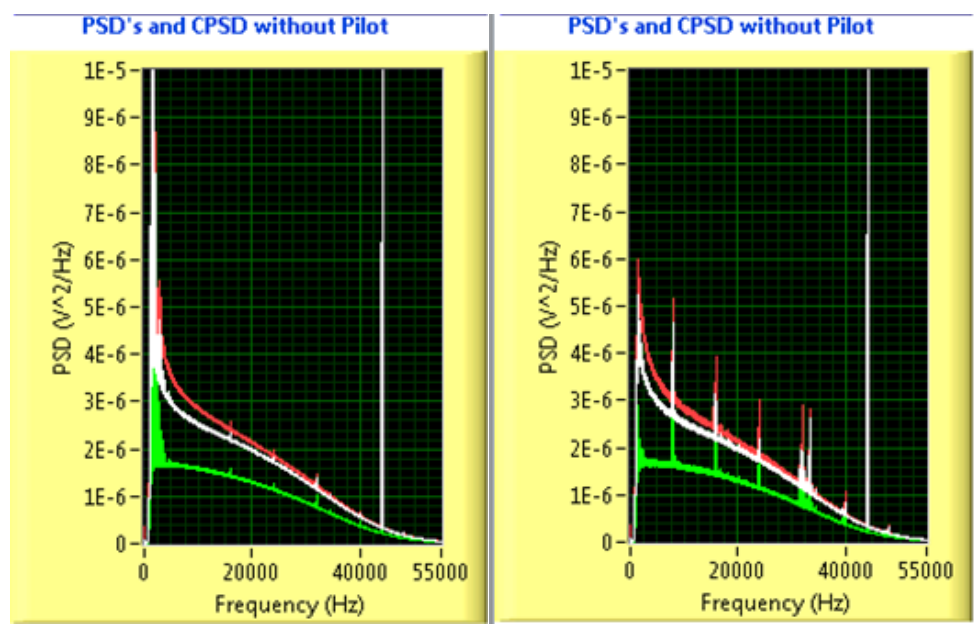

Cable $=0 \mathrm{~m}$, Error $=1.196 \mathrm{~K} \quad$ Cable $=5 \mathrm{~m}$, Error $=-0.208 \mathrm{~K}$

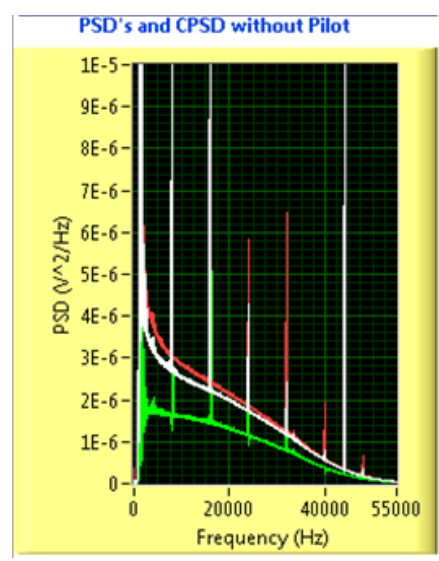

Cable $=10 \mathrm{~m}$, Error $=2.977 \mathrm{~K}$

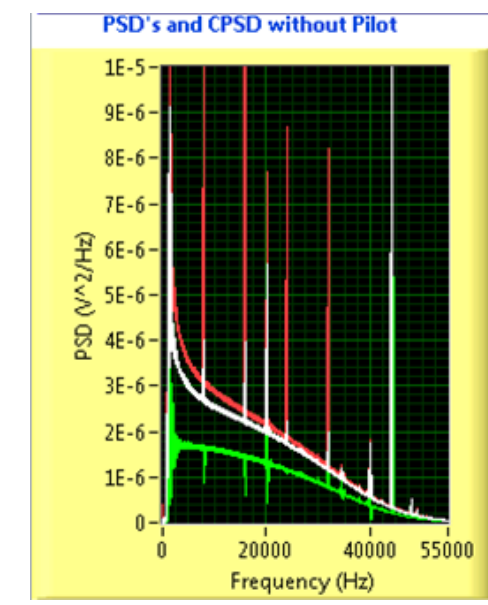

Cable $=25 \mathrm{~m}$, Error $=1.0 \mathrm{~K}$

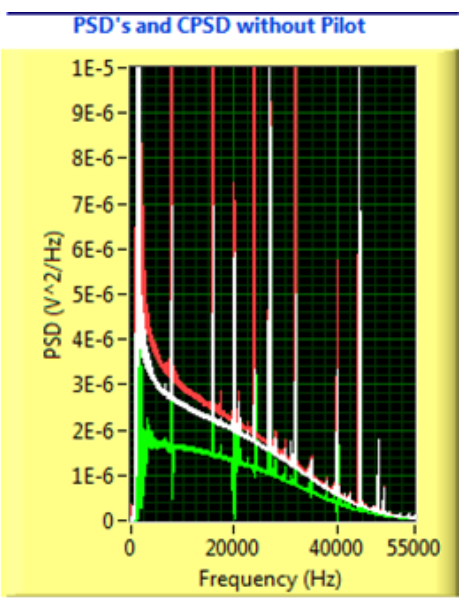

Cable $=25 \mathrm{~m}$, Error $=2.354 \mathrm{~K}$

Fig. 11. Data from Lab 35

Generally the spectra show the expected white noise from the differential channels as the smooth baseline of each of the curves. The plots in Figs. 10, 11, and 12 show the amplifier channels individually exhibit noise that increases as frequency decreases. This is as expected since the entire amplifier chain is configured as a low-pass filter. The very lowest 
frequencies below $5 \mathrm{kHz}$ exhibit sharp upturns due to harmonics from $60-\mathrm{Hz}$ interference. Since this is correlated in both channels, the cross-correlated signal (green), most of this is removed and is relatively flat at the lowest frequencies. It should be noted that the RTD, as shown in Fig. 5 always adds approximately $1 \mathrm{~m}$ to the stated cable length due to the cable permanently attached to the RTD itself.

The data in Fig. 10 indicates increasing EMI pickup above cable lengths of $5 \mathrm{~m}$. There is a strong interferer around $27 \mathrm{kHz}$ that is the high-voltage oscillator in the

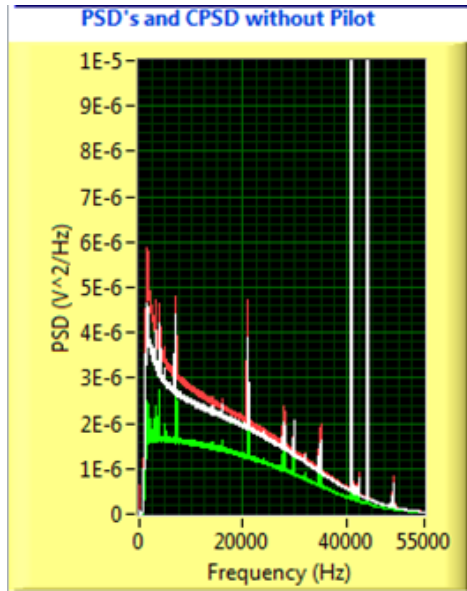

Cable $=0 \mathrm{~m}$, Error $=0 \mathrm{~K}$

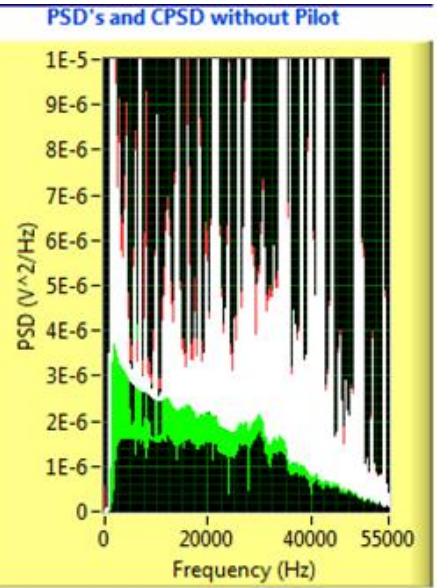

Cable $=5 \mathrm{~m}$, Error $=256 \mathrm{~K}$

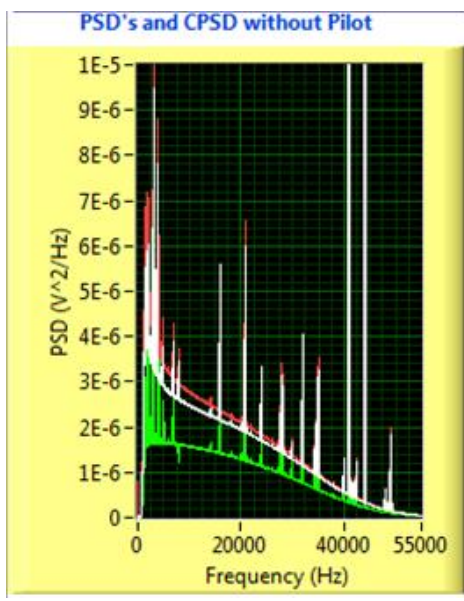

Cable $=10 \mathrm{~m}$, Error $=-0.448 \mathrm{~K}$

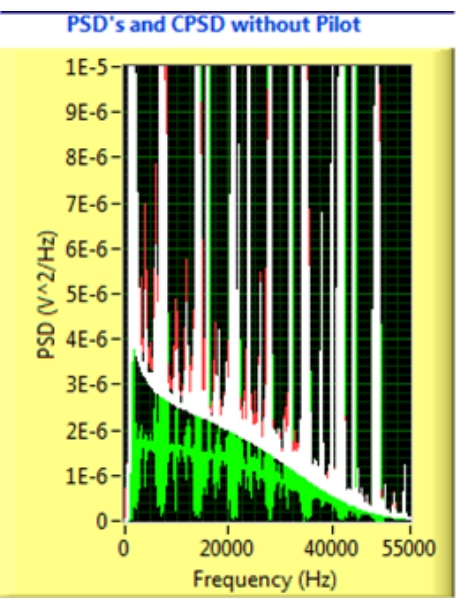

Cable $=10 \mathrm{~m}$, Error $=-0.911 \mathrm{~K}$

Fig. 12. Data from Cleanroom Chase

fluorescent-light ballasts overhead. In general, this is a relatively benign environment with the major wideband interferer exhibiting a peak around $20 \mathrm{kHz}$. The source of this noise has never clearly been identified. Also, there is a consistent interferer from the computer power supply that shows up around $42 \mathrm{kHz}$ on all these plots. This supply, if placed close to the input, can actually overload the system and cause dynamic-range issues. 
The Lab 35 data indicates a somewhat different environment. The 42-kHz interferer is present and is extremely narrow band. However, there is very little leakage from the light ballasts in this room. There is a hint of interference at approximately $8 \mathrm{kHz}$ that exhibits harmonics at the expected frequencies up to the cutoff of the system. None of these noise sources cause tremendous problems in calibration.

The cleanroom chase was a different story. The interferers are so numerous in the chase that they are hard to identify. In addition, since they are in close physical proximity to the RTD (a somewhat cramped room) they tend to inject more EMI onto the input. Fig. 12 is a clear example of what can be seen when the EMI overwhelms the system. The 5-m cable run shows an overwhelming amount of noise such that the measurement is completely unusable. Other runs, however, show a reasonable amount of resilience.

The important points shown by the data are:

1. Small amounts of EMI appear to have minor effects. There is always some effect but EMI starting and stopping appear to be manageable.

2. Longer cables pickup more EMI. The shorter cables obviously act less like antennae and will likely produce a better measurement if EMI sources such as motors and pumps are turning on and off.

3. Enough EMI will paralyze the system. As shown in Fig. 12, it is possible to inject a sufficient amount of EMI to make the measurement unusable, even with a shorter cable.

4. EMI will be averaged out only if it is not correlated between the two channels. Some of the interferers are visible on the red and white graphs but not on the green. Other interferers are visible on all. The uncorrelated signals are removed during the crosscorrelation operations. If EMI is injected onto the RTD itself and is correlated in both channels, the correlated portion appears to be part of the desired signal. This makes shielding extremely important.

\section{CONCLUSION}

The success of JNT depends to a large extent on the environment in to which it is deployed. From the testing discussed in this document the following assumptions were concluded. Shorter, well-shielded cables will perform better in a changing EMI environment. EMI can be managed by the signal processing but the environment into which a JNT system is deployed will need some level of analysis to ensure correct operation. If noise sources can be identified during the initial system calibration, they are more manageable.

We are presently exploring the possible use of sections of the spectrum instead of the complete, continuous spectrum. This would allow us to evaluate the spectrum and exclude some of the worst EMI interferers. 


\section{REFERENCES}

[1] Charles L. Britton, Jr., Michael Roberts, Nora D. Bull, Lou Qualls, and David E. Holcomb, "Johnson Noise Thermometry Requirements" ORNL/TM-2013/2, SMR/ICHMI/ORNL/TR-2013/01, January 2013

[2] Charles L. Britton, Jr., Michael Roberts, Nora D. Bull, , David E. Holcomb, and Richard T. Wood, "Johnson Noise Thermometry for Advanced Small Modular Reactors" ORNL/TM-2012/346, SMR/ICHMI/ORNL/TR-2012/01, September 2012

[3] Charles L. Britton, Jr., Nora D. Bull, Michael Roberts , "Amplifiers Module Prototype fir the Johnson Noise Thermometry System" ORNL/TM-2013/192, SMR/ICHMI/ORNL/TR-2012/03, May 2013

[4] Charles L. Britton, Jr., N. Dianne Bull Ezell, Michael Roberts, "Johnson Noise Thermometry Data-Acquisition Backend" ORNL/TM-2013/499, SMR/ICHMI/ORNL/TR-2013/06, October 2013 